Chirurg 2014 $\cdot 85: 96$

DOI 10.1007/s00104-013-2590-0

Online publiziert: 22. Januar 2014

๑) Springer-Verlag Berlin Heidelberg 2014

U.A. Dietz - C.T. Germer

Klinik und Poliklinik für Allgemein-, Viszeral-, Gefäß- und Kinderchirurgie, Universitätsklinikum Würzburg

\title{
Komplikationen der Hernienchirurgie
}

- das falsche Netz als Komplikationsursache,

- das Rezidiv als „sanfte“ Komplikation sowie

- die Diagnostik und Therapie des chronischen Schmerzes.

Mit diesen Themen stellen wir uns auch der Tatsache, dass unsere heutige Einschätzung der Realität durch zukünftige Erkenntnisse revidiert werden wird: Das war in der Vergangenheit und bleibt auch in Zukunft die Hoffnung und Faszination der Wissenschaft. Wir sind es unseren Patienten im Rahmen des Informationsgespräches schuldig, die immanente Vorläufigkeit unseres Tuns offenzulegen, auch wenn im angelsächsischen Raum wieder der kühne und naive Begriff der „cure of hernia" aufflackert. Der aktuellen Gesetzgebung folgend, sind wir nach der Operation verpflichtet, offen und unaufgefordert kleinere und größere Komplikationen zu kommunizierten und eine Perspektive des zu erwartenden Ergebnisses zu vermitteln. Der postmoderne Patient möchte als Gegenüber wahrgenommen werden und wünscht sich für seine Hernie einen "gechillten Experten“ und keinen „gestressten Spezialisten“. Und Komplikationen durch Stress? Noch schirmen Passion und Liebe zum Handwerk einen Teil des Druckes ab, doch hier ist noch einiges an Arbeit und gesundem Widerstand gegen den Leistungsdruck des Systems im Interesse der Patienten nötig.

Wir wünschen Ihnen mit den nachfolgenden Themen eine gute Zeit der Reflexion, der strategischen Hinterfragung und neue Freude bei der Versorgung Ihrer Hernienpatienten.

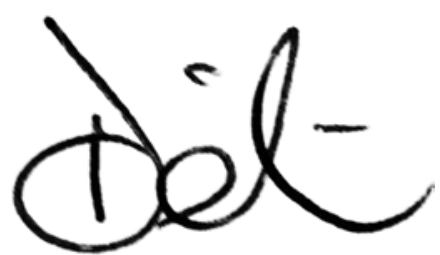

Prof. Dr. Dr. U.A. Dietz

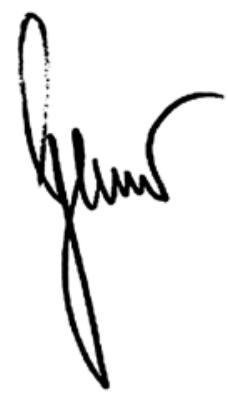

Prof. Dr. C.T. Germer

\section{Korrespondenzadresse}

Prof. Dr. Dr. (UFPR) U.A. Dietz

Klinik und Poliklinik für Allgemein-, Viszeral-, Gefäß- und Kinderchirurgie, Universitätsklinikum Würzburg, Oberdürrbacher Str. 6, 97080 Würzburg Dietz_U@ukw.de

Interessenkonflikt. U.A. Dietz und C.T. Germer geben an, dass kein Interessenkonflikt besteht.

\section{Literatur}

1. Kempe F (2006) Henry Kissinger, Interview vom 17. Juni. The Wall Street Journal

2. Heisenberg W (1976) Der Teil und das Ganze. Gespräche im Umkreis der Atomphysik. Piper, München Zürich, S 247 